\title{
UTILIZACIÓN DE MODELOS SIMULADORES PARA EL ENTRENAMIENTO DE ACCESOS VENOSOS CENTRALES: NUESTRA EXPERIENCIA
}

\author{
Alejandro M. Russo, Santiago Pose, Gabriela Aguiar, Gustavo J. Armand Ugón, \\ Eduardo A. Olivera
}

\author{
Departamento de Anatomía, Facultad de Medicina, Universidad de la República, \\ Montevideo, Uruguay
}

\section{RESUMEN}

Los modelos simuladores permiten representar un proceso mediante otro más simple, existiendo reportes de su utilización en las ciencias de la salud. El objetivo de este trabajo es evaluar el rendimiento de modelos simuladores en el entrenamiento de los accesos venosos centrales. Durante 2 cursos organizados por la Asociación Uruguaya de Anatomía, se utilizaron modelos simuladores para el entrenamiento en la punción venosa central. Se consultó, por vía electrónica, a 60 participantes en una encuesta de opinión de 4 declaraciones utilizando una escala de Likert. Se obtuvieron 57 (95\%) cuestionarios que se procesaron en su totalidad, 53 (93\%) estuvieron fuertemente de acuerdo o de acuerdo que el modelo simulador es útil para el entrenamiento de la punción venosa. Respecto a la importancia del modelo simulador en el manejo del material de punción, 57 $(100 \%)$ de los participantes estuvieron fuertemente de acuerdo o de acuerdo. Sobre si los modelos simuladores debían ser utilizados sistemáticamente en el entrenamiento de punción venosa, 50 (88\%) estuvieron fuertemente de acuerdo o de acuerdo. En cuanto al empleo de los modelos similares para el entrenamiento de otras maniobras, 52 (91\%) consideraron fuertemente de acuerdo o de acuerdo. Los modelos simuladores fueron considerados de utilidad por los participantes. Concluimos que la utilización de simuladores es de utilidad en la enseñanza de punciones venosas y debería propenderse su uso. Por tanto debemos doblar nuestro esfuerzo tanto en la producción como en la utilización de los modelos simuladores.

Palabras clave: simulación, punción venosa central, maniobras invasivas, anatomía clínica.

\begin{abstract}
Simulators allow the representation of a complex process as a simpler one, analyzing its characteristics. The use of simulators in the medical sciences has been reported. The aim of this article is to evaluate the utility of simulators in the training for central venous access. The "Asociación Uruguaya de Anatomía" organized 2 courses in which simulators were used in the training of central venous access. Sixty (60) participants of these courses were electronically consulted at a survey of 4 declarations using a Likert's scale, $57(95 \%)$ interviews were obtained and completely processed; $53(93 \%)$ of the participants strongly agreed or agreed that the simulator was useful in the training of central venous access. Regarding the importance of the simulator in manipulating the materials, $57(100 \%)$ of the participants strongly agreed or agreed with the statement. When asked if the simulator should be systematically used in the training of central venous access, 50 (88\%) strongly agreed or agreed; 52 (91\%) of the participants strongly agreed or agreed that the simulators should be used in the training of other maneuvers. Simulators were considered useful in the maneuver training by the participants of both courses. We conclude that simulators were found useful in the training of central venous access and its usage should be encouraged. Thus we should double our efforts in both production and usage of simulators.
\end{abstract}

Key words: simulation, central venous access, invasive procedures, clinical anatomy.

* Correspondencia a: Dr. Alejandro Russo. Vazquez Ledesma 3021, Montevideo, Uruguay. aleru86@gmail.com

Recibido: 4 de febrero, 2014. Revisado: 18 de febrero, 2014. Accepted: 27 de febrero, 2014. 


\section{INTRODUCCIÓN}

Uno de los recursos más novedosos utilizado en las ciencias de la Salud son los modelos simuladores (MS) (Mendoza et al, 2009). La simulación se reconoce como una herramienta docente capaz de solventar limitaciones de la metodología docente tradicional y está bien establecida en la formación de otros profesionales como pilotos de aeronáutica, bomberos y trabajadores de centrales nucleares (Inssenber et al, 1999). Las ciencias de la salud se consideran uno de los campos en los que la simulación tiene más aplicación para la formación tanto del pre como del post-grado, para la formación continúa y adquirir las destrezas suficientes para la realización de diversas maniobras como ser la punción venosa, arterial, intubación oro-traqueal, estudios endoscópicos, entre otros (Lateef, 2010). Los MS tienen como fundamento brindar al alumno la oportunidad de práctica constante de destrezas psicomotrices mientras se familiariza con instrumentos y equipos, y al mismo tiempo gana experiencia en el reconocimiento de problemas y en el desarrollo de toma de decisiones, así como en el perfeccionamiento de técnicas y procedimientos. La gran ventaja que presentan estos MS es que preparan al alumno para una situación real. Esta preparación es sin riesgo para el paciente, además le permiten al participante autoevaluarse e ir una y otra vez sobre sus errores para llegar a las correcciones necesarias para lograr la técnica, hecho imposible de hacer en el paciente sin riesgos de morbilidad (Ziv et al, 2006).

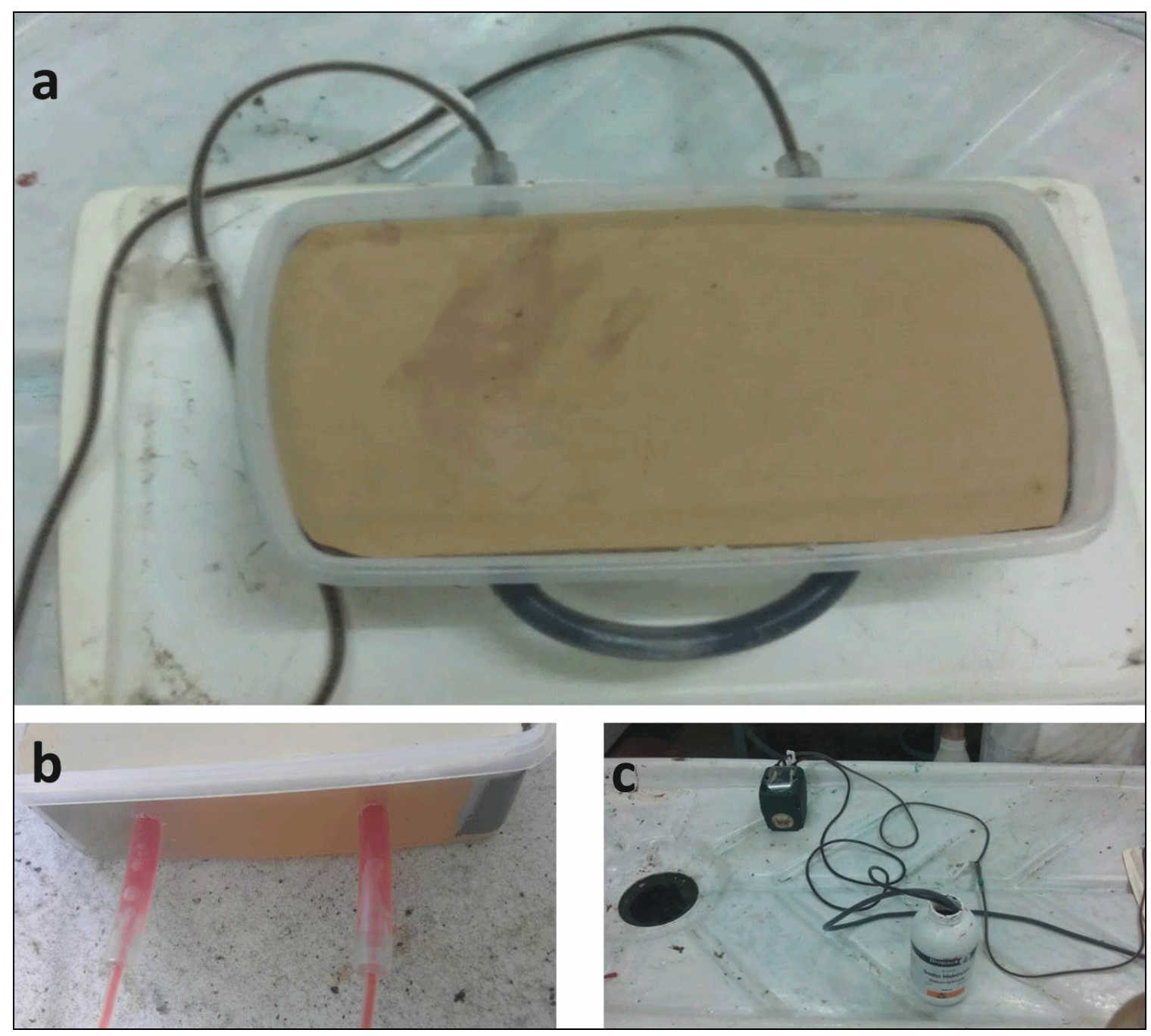

Figura 1 - a. Vista superior del MS. Se visualiza el envase de plástico relleno de gelatina. En su interior el tubo de silicona doblado en "U". b. Vista posterior del MS. Se visualiza la conexión del tubo de silicona con la bomba pulsátil, sirviendo uno de entrada y el otro de salida del líquido coloreado. c. Se visualiza la bomba pulsátil que permite la circulación del líquido coloreado por el MS. 
Durante el año 2012 se llevaron a cabo en el Departamento de Anatomía de la Facultad de Medicina de Montevideo (Universidad de la República), dos actividades de entrenamiento de accesos venosos centrales; por una parte el curso titulado "Bases anatómicas para la realización de las vías venosas centrales" organizado por la Asociación Uruguaya de Anatomía (AUA), y por otra parte el curso precongreso del "XVI Congreso Uruguayo de Emergencia, Trauma y Desastres" organizado por la AUA en conjunto con la Sociedad Uruguaya de Emergencia y Trauma. Ambos cursos estaban dirigidos a pre-grados avanzados en la carrera de Doctor en Medicina y postgrados.

En ambas actividades se tuvo en demostración un MS realizado por los autores de este trabajo el cual el participante puede similar un acceso venoso central. Esto nos permitió realizar un estudio cuyo objetivo fue determinar la opinión de los participantes de los mencionados cursos sobre la utilización de esta metodología.

\section{MATERIALES Y MÉTODO}

Durante ambos cursos, el participante dispuso del MS y del kit completo para realizar el acceso venoso simulado de acuerdo a la técnica de Seldinger. (Imigo et al, 2011) El MS utilizado consiste de un envase de plástico, con gelatina de uso cotidiano en su interior. Inmerso en la misma se incluye un tubo de silicona de $1 \mathrm{~cm}$ de diámetro que se dobla en "U" para poder tener dos sitios de acceso posibles en un solo MS. Ambos extremos de dicho tubo se conectan a una bomba pulsátil que permite la circulación del líquido coloreado (Fig. 1). Si la punción es satisfactoria se recupera líquido coloreado que simula la sangre al puncionar la vena. A continuación, el participante coloca la guía para finalmente cateterizar el tubo que emula la vena. Encomendamos al lector el video del funcionamiento del MS utilizado en el presente trabajo. (Russo et al, 2012).

Una vez finalizados los cursos, se contactaron por vía electrónica 60 (69\%) participantes al azar de un total de 87 . El grupo estaba formado por $45(75 \%)$ estudiantes de pre-grado de la carrera Doctor en medicina y 15 (25\%) pos-grados. Se les envió mediante correo electrónico una breve encuesta anónima de opinión de 4 declaraciones relacionadas con la experiencia vivida en la sesión con el MS, utilizando una escala de Likert (Tabla 1). El $100 \%$ de los participantes $(n=60)$ fue consultado previo al envío de la encuesta si deseaba formar parte de este trabajo, al aceptar, se les enviaba el formulario.

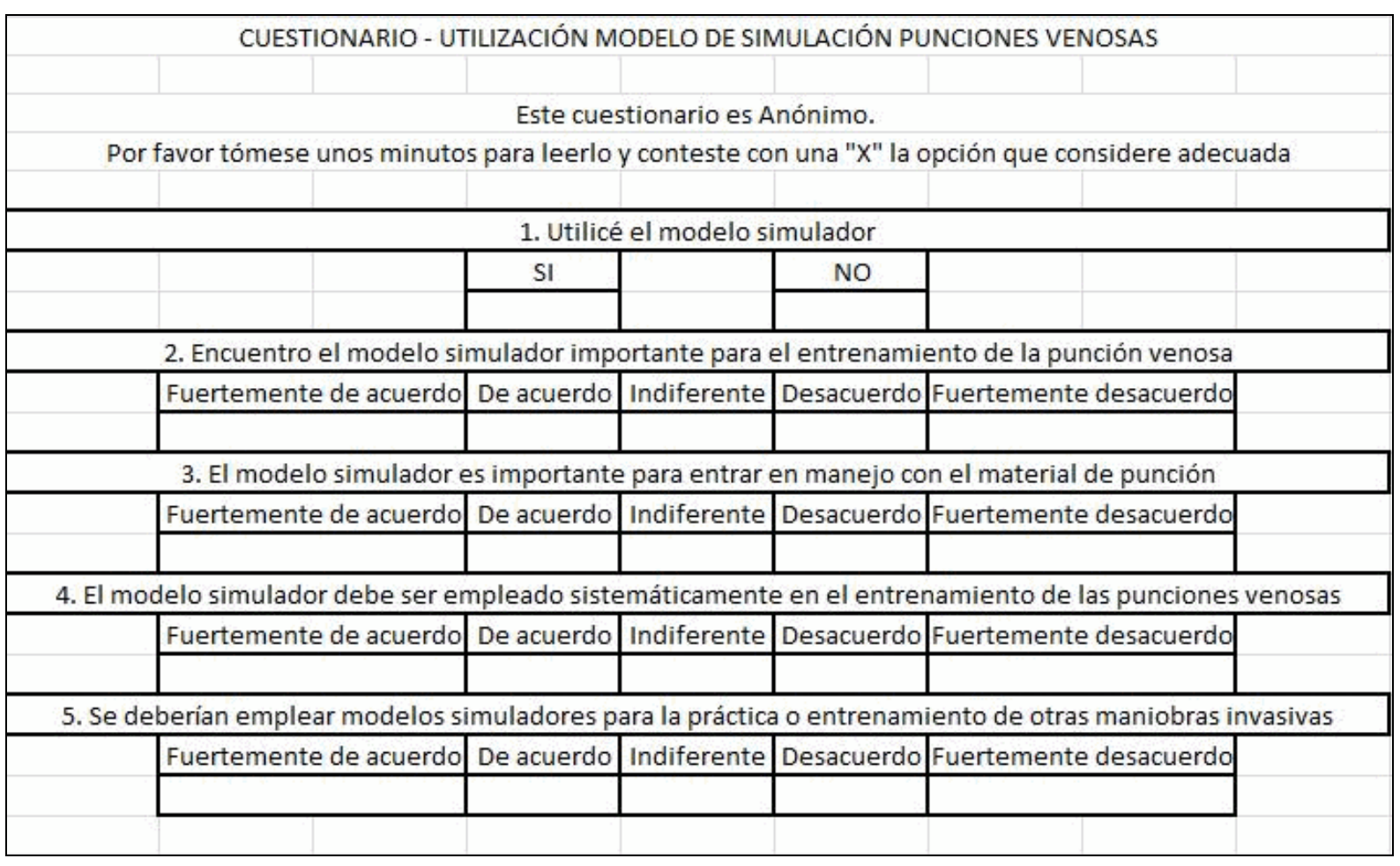

Tabla 1. Formulario enviado a los participantes de los cursos 


\section{RESULTADOS}

De los 60 cuestionarios enviados por vía electrónica fueron recibidos 57 (95\%), los mismos estaban contestados en su totalidad. Los resultados completos se expresan en la Figura 2. Destacamos que de la totalidad de los cuestionarios recibidos, el 100\% $(n=57)$ utilizaron el MS. De éstos, 53 (95\%) participantes estuvieron fuertemente de acuerdo o de acuerdo con que el MS es útil para el entrenamiento de la punción venosa. Cuando fueron preguntados por la importancia que tiene el MS como medio de comprensión del manejo del material de punción, $57(100 \%)$ de los participantes estuvieron fuertemente de acuerdo o de acuerdo. Respecto a la utilización sistemática de los MS para el entrenamiento de la punción venosa, 50 (88\%) estuvieron fuertemente de acuerdo o de acuerdo. Por último, 52 (91\%) consideraron fuertemente de acuerdo o de acuerdo, que se debe emplear la utilización de similares MS para el entrenamiento de otras maniobras invasivas.

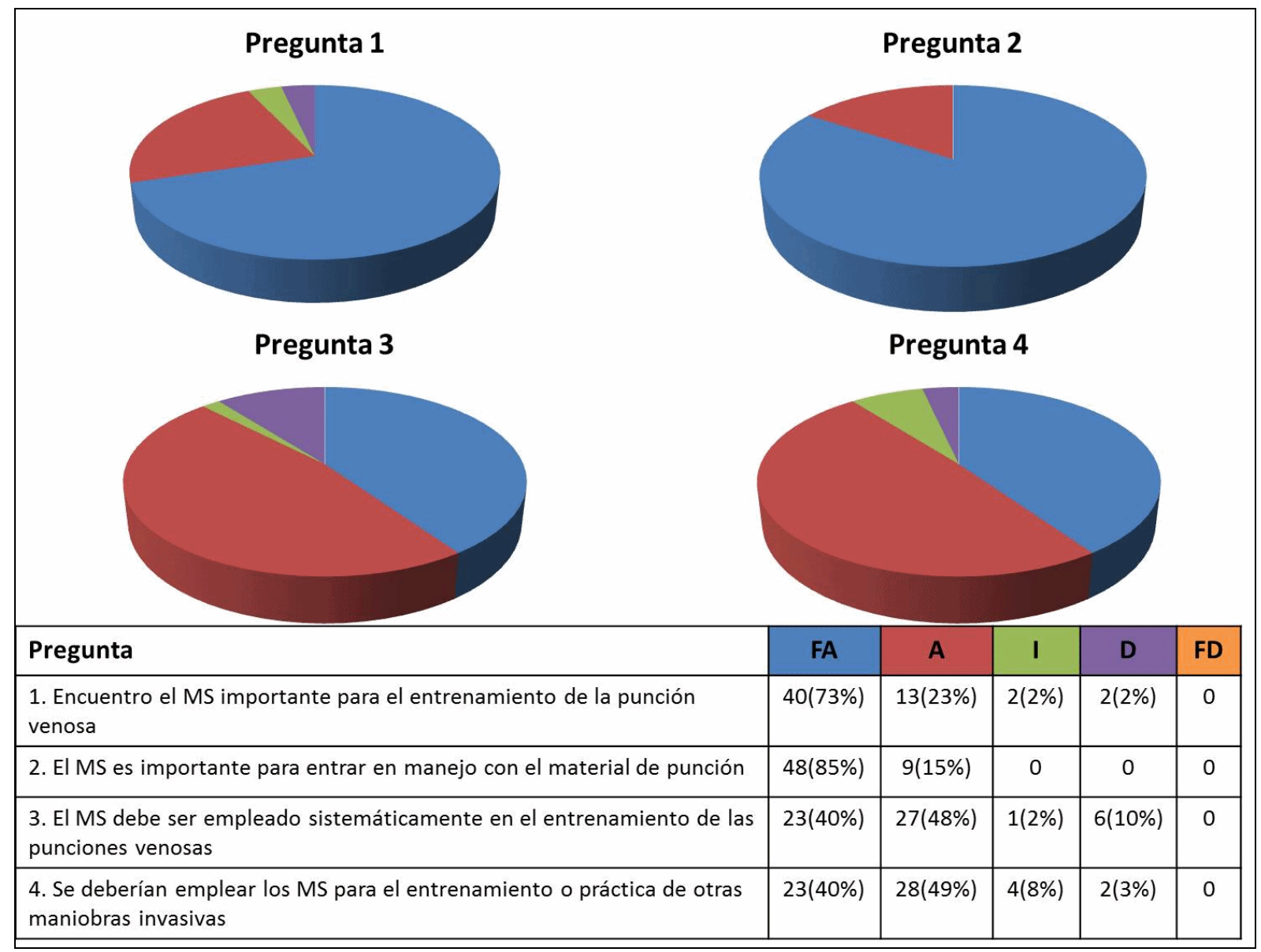

Figura 2 - Esquema de los resultados obtenidos. En la tabla inferior se objetivan los resultados de acuerdo a las preguntas realizadas, resultado absoluto y porcentaje. Por encima los gráficos obtenidos a partir de cada pregunta. FA Fuertemente de acuerdo (color azul), A: De acuerdo (color rojo), I: Indiferente (color verde), D: Desacuerdo (color violeta), FD: Fuertemente desacuerdo (color naranja).

\section{DISCUSIÓN}

Presentamos la primera experiencia en la utilización de MS en el Departamento de Anatomía, de la Universidad de la República, Montevideo, Uruguay. Al igual que otros autores
(Mendoza et al, 2009), consideramos de interés investigar el valor de este método de enseñanza, sobre todo si se considera la progresiva reducción de situaciones clínicas reales, tanto en hospitales como en el ambiente ambulatorio, utilizables para aprender. 
Existen reportes de otras experiencias en la construcción de MS para el entrenamiento de los accesos venosos centrales, encontrando fundamental rédito simulando la técnica de punción bajo visión directa ultrasonográfica (Kendall y Faragher, 2007). Otros investigadores han obtenido resultados prometedores utilizando MS, simulando otras situaciones. Ejemplo de esto es el grupo de Mendoza et al (2009), utilizando el MS Emergency Care Simulator (ECS) encontrando diferencias estadísticamente significativas entre un grupo de estudio que utilizó el MS y un grupo control. Otro ejemplo de esto es la experiencia aportada por Gupta et al (2008), en la que previo a utilizar un MS, que también simulaba situaciones de medicina de emergencia e incluía escenarios médicos, quirúrgicos, traumatológicos y mixtos; se entregó un formulario pre-test, y luego de finalizada la experiencia el mismo formulario a modo de pos test, encontrando diferencias sustanciales entre los resultados del pre y post-test.

Ahora bien, el buen rendimiento de los MS, ha llevado a autores a afirmar que la simulación es el medio por el que las habilidades clínicas se aprenderán lejos del paciente (Binstadt et al, 2007). En nuestra formación tanto anatómica como clínica, fundamentalmente práctica, discrepamos con este concepto. Es nuestra opinión que el MS debe ser un complemento, una herramienta más que enriquezca el aprendizaje pero que de ningún modo sea un reemplazo de otras metodologías. En consonancia, durante los cursos organizados por la AUA anteriormente citados, los participantes no solo se enfrentaban al MS, sino que en otras estaciones contaban con disecciones anatómicas de cuello y muslo para reconocer los reparos anatómicos fundamentales y luego practicar la punción venosa en los mismos. Esto último, se encuentra en sintonía con las recomendaciones basadas en la evidencia para el entrenamiento de los accesos venosos centrales (Moureau et al, 2013), en donde el conocimiento anatómico es considerado fundamental.

De nuestra experiencia se desprende que la amplia mayoría de los encuestados coincidieron en la utilidad de los MS para practicar la punción venosa, así como también para ganar práctica en el manejo de los instrumentos del kit para punción según la técnica de Seldinger. Por otro lado, una gran parte de los participantes opinaron que se debe emplear el uso de los MS para aquellos pre y post-grados interesados en aprender la técnica de la punción venosa central. Es más, también hubo una amplia mayoría de personas totalmente de acuerdo en que se debe ampliar el terreno de uso de los MS para otras maniobras invasivas. Todo esto nos obliga a doblegar nuestros esfuerzos en la producción así como en la utilización de los MS. Por tanto consideramos a los MS como una herramienta útil, complementaria a los métodos tradicionales de enseñanza.

En conclusión, los resultados obtenidos nos sugieren que la incorporación de MS en el entrenamiento de los accesos venosos centrales puede llegar a ser de utilidad para los participantes.

\section{BIBLIOGRAFÍA}

Binstadt ES, Walls RM, White BA, Nadel ES, Takayesu JK, Barker TD, Nelson SJ, Pozner CN. 2007 A comprehensive medical simulation education curriculum for emergency medicine residents. Ann Emerg Med, 49: 495-507.

Gupta A, Pecler B, Schoken D. 2008 Introducing of hi-fidelity simulation techniques as an ideal teaching tool for upcoming emergency medicine and trauma residency programs in India. $\mathrm{J}$ Emerg Trauma Shock 1: 15-18.

Imigo FG, Elgueta AC, Castillo FE, Celedón EL, Fonfach CZ, Lavanderos FJ, Mansilla ES. 2011 Accesos Venosos Centrales. Cuad Cir, 25: 5258.

Issenber SB, Mc Gaghie WC, Hart IR, Mayer KW, Felner JM, Petrusa ER, Waugh RA, Brown $D D$, Safford RR, Gessner IH, Gordon DL, Ewy $G A .1999$ Simulation technology for health care professional skills training and assessment. JAMA, 282: 861-66.

Kendall JL, Faragher JP. 2007 Ultrasound-guided central venous access: a homemade phantom for simulation. CJEM, 9: 371-73.

Lateef F. 2010 Simulating-based learning: just like the real thing. J Emerg Trauma Shock, 3: 348-352.

Mendoza L, Herskovic P, Vásquez A, Quevedo F. 2009 Uso de modelos simuladores como metodología docente para la carrera de Medicina. Rev. Hosp. Clín. Univ Chile, 20: 35559.

Moureau

$N$, Lamperti M, Kelly LJ, Dawson R, Elbarbary M, Van Boxtel AJ, Pittiruti M. 2013 Evidence-based consensus on the insertion of central venous access devices: definition of minimal requirements for training. $\mathrm{Br} J$ Anaesth, 110: 347-56.

Russo A, Pose S, Aguiar G, Armand Ugón G, Olivera E. 2012 Video - funcionamiento del MS. Disponible en: https://docs.google.com/file/ d/0B9iW21vRBZ4rc3p1YVIrM1JKSmM/edit. Visitado el día 31/01/14. 
Ziv A, Erez D, Munz Y, Vardi A, Barsuk D, Levine I, Benita S, Rubin O, Berkenstadt H. 2006 The Israel Center For Medical Simulation: A paradigm for cultural change in medical education. Acad Med, 81: 1091-97. 